\title{
La troisième révolution touristique
}

\author{
Philippe Violier
}

\section{OpenEdition}

\section{Journals}

Édition électronique

URL : http://journals.openedition.org/tourisme/1256

DOI : 10.4000/tourisme.1256

ISSN : 2492-7503

\section{Éditeur}

Éditions touristiques européennes

\section{Référence électronique}

Philippe Violier, « La troisième révolution touristique », Mondes du Tourisme [En ligne], Hors-série | 2016 mis en ligne le 01 septembre 2016, consulté le 19 avril 2019. URL : http://journals.openedition.org/ tourisme/1256 ; DOI : 10.4000/tourisme.1256

Ce document a été généré automatiquement le 19 avril 2019

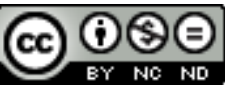

Mondes du tourisme est mis à disposition selon les termes de la licence Creative Commons Attribution - Pas d'Utilisation Commerciale - Pas de Modification 4.0 International. 


\title{
La troisième révolution touristique
}

\author{
Philippe Violier
}

1 Tous les chercheurs et les praticiens s'accordent pour considérer que le tourisme subit dans la période actuelle des mutations importantes depuis l'arrivée d'internet à l'explosion du tourisme dans les sociétés émergentes. Nous nous référons ici au concept de tourisme élaboré par l'équipe Mit $(2002,2005,2011)$ qui distingue cette pratique sociale d'autres mobilités comme les voyages d'affaires ou les pèlerinages. Le sens du déplacement, l'inscription plus ou moins importante dans un espace de liberté, la prise en charge par les individus... de nombreux éléments permettent de fonder cette distinction (Stock, 2003 ; Knafou et Stock 2003). Or depuis son invention au XviII ${ }^{e}$ siècle, le tourisme a déjà connu plusieurs transformations majeures sans que la finalité du système, la recréation, n'ait été remise en cause. Les travaux des historiens, Marc Boyer (1996) et Laurent Tissot (2001) notamment, permettent de distinguer deux grandes périodes. Le premier a mis en évidence l'invention du tourisme, contemporaine de la révolution industrielle $\mathrm{du} \mathrm{xVIII}^{\mathrm{e}}$ siècle. Le second a montré comment plusieurs événements contemporains, l'invention du guide touristique, l'avènement du tour-operating, le développement du réseau ferré font système au moment du passage au tourisme du grand nombre, alors que la révolution industrielle produit ses premiers effets bénéfiques au milieu $\mathrm{du} \mathrm{xIX}^{\mathrm{e}}$ siècle (hausse $\mathrm{du}$ pouvoir d'achat, premiers congés payés...). Les événements en cours qui affectent le système tourisme donnent à penser qu'une troisième phase est engagée, même si son actualité en rend la lecture plus difficile.

2 Nous manquons cependant d'un cadre conceptuel pour appréhender ces évolutions et pour intégrer les changements actuels. Cet article se propose de contribuer à le construire en s'appuyant sur le concept de système sociotechnique et en l'enrichissant de dimensions nouvelles, spatiales notamment. Auparavant, nous discuterons les thèses sur la fin du tourisme, qu'il convient d'écarter avant d'analyser comment le tourisme en tant que pratique sociale se renouvelle aujourd'hui. 


\section{Le tourisme a une histoire}

3 L'historicité du tourisme mérite d'être affirmée au départ de cette analyse sur les temporalités de cette pratique sociale. En effet certains auteurs la nient en considérant que le tourisme a toujours existé. Nous nous appuierons sur les travaux d'historiens qui étayent la thèse d'une invention du tourisme. Ensuite d'autres auteurs évoquent une fin du tourisme, auquel cas la question de son devenir ne se pose plus.

\section{Soit un début}

4 Marc Boyer (1996) a notamment montré que le tourisme a été inventé en même temps que la révolution industrielle. Cette approche contribue notamment à distinguer le tourisme des voyages ${ }^{1}$ par la mise en évidence d'une finalité nouvelle pour ces mobilités, la recréation (équipe Mit, 2002). La relation avec la révolution industrielle s'exprime doublement. D'une part, cette dernière crée les conditions d'un nouveau partage du temps. Au sein du temps contraint, l'accroissement des « actions routinières » (Dunning et Elias, 1994, p. 134) rend nécessaire en contrepartie le développement du hors-quotidien pendant lequel l'individu peut se reconstruire. Ce va-et-vient avait déjà été abordé par Enzensberger (1958) et Jafar Jafari (1988). D'autre part, le mode de production industriel crée les conditions économiques, techniques et sociales à un développement des mobilités du temps libre. Ces transformations s'effectuent progressivement. De même que les historiens repèrent des formes de proto-industrie, des formes de prétourisme apparaissent dès le $\mathrm{XVI}^{\mathrm{e}}$ siècle comme le Grand Tour, ce voyage initiatique qui conduisait notamment, en deux ou trois années, les aristocrates de l'Europe vers les ruines de Rome, la villégiature des grands propriétaires ou les loisirs urbains. Il n'empêche que l'invention du tourisme constitue bien une rupture qui justifie l'usage du mot révolution.

\section{Et peut-être une fin ?}

5 Mais si le tourisme a un début, il est légitime de lui envisager une fin. Serait-elle atteinte aujourd'hui ou en passe de l'être? Trois postures différentes s'inscrivent dans cette perspective. L'une s'appuie sur le constat que des lieux créés par le tourisme ne sont plus aujourd'hui touristiques au sens où ils ne sont plus fréquentés seulement de manière temporaire par des individus en quête de recréation mais qu'ils sont également, voire essentiellement, habités de manière sédentaire. Cette thèse est notamment défendue par John Urry (1995) qui appuie sa démonstration sur la dynamique des stations littorales anglaises. Nous partageons l'analyse d'Alain Girard (2013) selon laquelle il s'agit là d'une erreur de perspective et d'une généralisation au monde d'une analyse pertinente de l'évolution de lieux particuliers. En effet, d'une part, l'accroissement de la mobilité des individus a étendu l'espace quotidien et a repoussé les marges du hors-quotidien. D'autre part, cette même dynamique a rendu possible l'habitat polytopique (Stock, 2006), soit la possibilité dans une société à individus mobiles d'exercer des mobilités entre plusieurs lieux inscrits dans le quotidien au sens où ces déplacements sont fréquents, répétés et peu différenciés du point de vue des pratiques. Nous voudrions insister sur ce dernier point qui semble absent d'analyses qui questionnent la place des résidences secondaires par rapport au tourisme. Or ce n'est pas l'analyse fine de l'objet qui nous éclaire mais bien 
ce que les individus font avec cet objet. Dès lors mettre en œuvre des pratiques du quotidien dans une résidence l'intègre dans l'espace polytopique du quotidien au-delà de la localisation dans un lieu qui a été touristique à un moment de son histoire ou qui l'est encore pour d'autres individus. Il devient alors possible de modéliser cette dynamique de la dichotomie quotidien-hors quotidien (figure 1). La proposition sous-jacente à cette modélisation considère que l'accroissement de la mobilité au sein des sociétés avancées techniquement induit une extension de l'espace quotidien des individus qui s'approprient par leurs compétences spatiales des étendues plus vastes et discontinues. Ainsi un espace comme les côtes de Normandie est-il passé, pour les résidents aisés de l'Ouest parisien, d'un lieu du hors-quotidien au XIX ${ }^{e}$ siècle, seulement fréquenté de manière saisonnière, à un espace accessible toute l'année, pendant les fins de semaine notamment.

Fig. 1 : dynamique de la dichotomie quotidien/hors quotidien

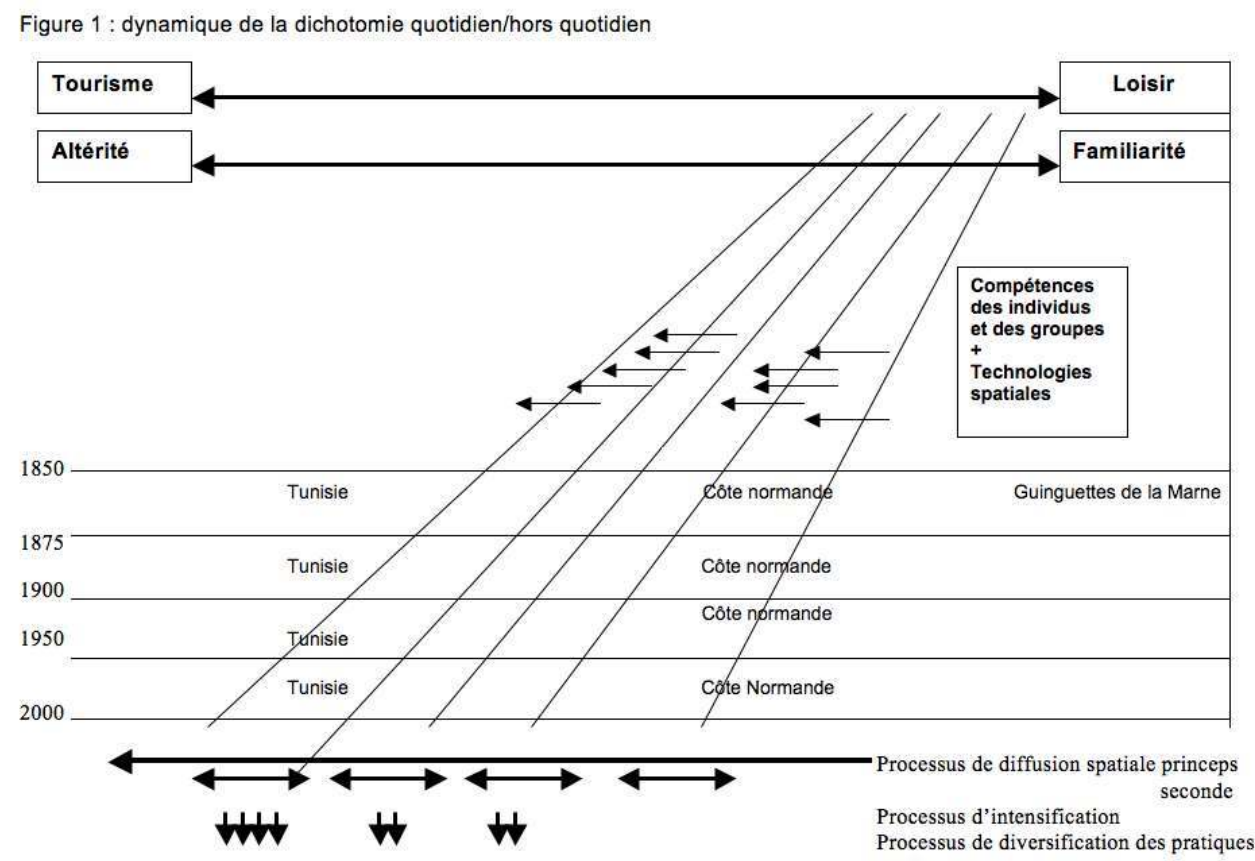

(c) Violier, 2016

6 La seconde thèse des tenants de la fin du tourisme se veut plus globale. Notons tout d'abord qu'il nous semble curieux d'annoncer la fin d'une réalité sociale au moment où elle devient mondiale. Nous reviendrons sur cette question qui constitue de notre point de vue une caractéristique fondamentale du tourisme d'aujourd'hui. Ensuite, le déni du fait touristique qui accompagne le faire-part ne contribue pas à crédibiliser la thèse. Nous relevons notamment, dans une introduction très contournée, que Philippe Bourdeau (2013) n'hésite pas à affirmer, sans étayer cette affirmation par une quelconque mention de source, que le tourisme ne concerne que $4 \%$ de la population mondiale. Or sur quoi est fondée cette estimation? Probablement sur les statistiques de l'Organisation mondiale du tourisme (OMT). Or, cette institution estime à plus d'un milliard le nombre des «touristes »: de fait 1 milliard sur 7, cela fait $14 \%$ et non $4 \%$. Par ailleurs, et surtout car la coquille n'est pas impossible, cette estimation est sans doute trompeuse. Tout d'abord parce que la définition très large mobilisée par l'OMT conduit à des surestimations : il s'agit davantage de voyageurs que de touristes. Une donnée produite par la même 
organisation estime que $52 \%$ des voyageurs circulent pour la recréation, $27 \%$ pour rendre visite à des parents et amis et $17 \%$ pour affaires ( $7 \%$ ne précisent pas). Mais, et surtout, cette assertion ignore complètement le tourisme intérieur, beaucoup plus massif. Notons par exemple que, à l'exception des États dotés d'un territoire restreint, dans tous les pays du monde les effectifs touristiques qui circulent à l'intérieur des frontières sont plus nombreux que ceux qui les franchissent (Violier, 2013). Nous pourrions éventuellement considérer que les mobilités à l'intérieur de l'espace national ne relèvent plus du tourisme mais des loisirs, en raison notamment du faible gradient d'altérité qui subsiste au sein d'un État travaillé depuis des siècles par les processus de centralisation, rejoignant en cela la thèse précédente (fig. 1). Mais cette posture n'a pas de sens pour des États continentaux comme l'Inde (Sacareau, 2011), la Chine (Taunay, 2011), l'Indonésie (Cabasset-Semedo et alii, 2010 ; Parantika, 2015 ; Parantika et Pickel-Chevalier, 2015) ou le Brésil qui fournissent des contingents très nombreux de touristes mobiles à l'intérieur d'un vaste territoire au sein duquel de profondes disparités culturelles demeurent. Enfin, et plus fondamentalement, le catastrophisme accompagne les études sur le tourisme depuis leur émergence. Rappelons notamment Gormsen annonçant le déclin de la Côte d'Azur (1981). Une partie des intellectuels a toujours éprouvé des difficultés à penser le tourisme et en annonce régulièrement la fin. La mobilisation d'une littérature hétéroclite (notamment par Bourdeau, 2013) au sein de laquelle les travaux scientifiques côtoient, pour appuyer la démonstration, les brûlots idéologiques constitue un des signes de cette phobie. En quelque sorte, pour ces auteurs, il est temps que le tourisme, affublé de tous les maux, disparaisse. Or le tourisme est une pratique sociale qui existe, qui a le sens que les individus et les sociétés lui donnent, et le rôle des scientifiques est de comprendre, non de juger.

Enfin une troisième thèse ne fonctionne pas dans le réel. Elle n'annonce pas la fin du tourisme comme un constat mais aboutit au même souhait de sa disparition au nom du développement durable. En effet les mobilités, dont le tourisme, induisent nécessairement une consommation d'énergie fossile et pèsent donc sur les ressources non renouvelables tout en contribuant aux rejets de gaz à effets de serre. Cependant, les critiques considèrent que le tourisme serait moins légitime à polluer que d'autres activités car il serait futile, ce qui, de la part d'un courant qui fustige par ailleurs le productivisme, étonne. Du coup des auteurs proposent «le tourisme virtuel» comme alternative (notamment Dewailly, 1997 ; Babou et Callot, 2007, 2012). Les performances de la technique nous dispenseraient des voyages. Au-delà, et comme le rappelle Julien Gardaix (2013), rien ne remplace l'expérience vécue par l'individu in situ. Également, les images ont toujours contribué au développement du tourisme (équipe Mit, 2005) et la croissance continue des flux médiatiques s'est accompagnée de celle des déplacements, si bien que la relation entre la communication et les mobilités fonctionne exactement à l'inverse : plus il y a d'images, plus il y a de voyages.

8 Le tourisme n'explore donc pas sa fin. Dès lors nous pouvons poser la question de sa périodisation.

\section{Le tourisme comme système sociotechnique}

9 Si le tourisme a bien une historicité au sens d'une émergence datée, cette invention n'a pas accouché d'un système définitivement stable. Nous nous proposons donc d'en définir les étapes. 


\section{La dynamique du tourisme} font ensuite système, au sens où des modalités différentes sont liées et évoluent dans une même période. L'historien souligne ainsi que dans un intervalle de temps relativement restreint, une cinquantaine d'années, le guide touristique est inventé, vers 1830, comme genre littéraire et supplante le récit de voyage, les touristes s'approprient le chemin de fer vers 1850 pour remplacer la traction hippomobile, l'hôtel confortable remplace l'auberge infâme, la station se déploie vers 1850 également à la place de la villégiature (équipe Mit, 2005) et le tour-opérateur supplante l'initiative individuelle à la même période. Ajoutons aussi que les congés payés constituent l'innovation sociale et l'avènement $\mathrm{du}$ suffrage universel l'avancée politique majeure qui encadrent ces mutations techniques. Toutes ces innovations caractérisent le passage du tourisme réservé à une minorité d'aristocrates et de grands bourgeois rentiers à un tourisme du grand nombre, qui préfigure le tourisme de masse - défini comme l'accès au tourisme du plus grand nombre au sein d'une société - qui s'épanouira entre les années 1920 et 1950 aux États-Unis et les années 1960 et 1970 en Europe. En effet, le guide va diffuser une information plus rationnelle à des touristes plus nombreux qui ne peuvent plus compter sur les réseaux familiaux pour être hébergés, qui ont besoin de moyens de transport plus efficaces car ils ne peuvent comme les rentiers prendre leur temps, et qui vont être soutenus par des professionnels capables d'assembler les éléments du système productif du tourisme (transport, hébergement, restauration, loisir). Laurent Tissot (2001) expose ainsi comment Thomas Cook a dû convaincre les compagnies françaises de chemin de fer de vendre à l'avance des billets, d'en coordonner les horaires, et a dû séduire les hôteliers suisses afin d'assurer aux touristes britanniques le breakfast en lieu et place du petitdéjeuner autochtone. Ces travaux nous informent donc de ce que la mutation d'un système à l'autre ne se produit pas à un moment bien identifié, voire à une date précise, mais se comprend par la mise en relation de plusieurs faits dont les significations s'éclairent les unes par les autres. En ce sens, le mot révolution est davantage à comprendre dans l'acception donnée une période, plutôt qu'à une date clé.

\section{La théorie des paradigmes sociotechniques}

11 Dès lors il s'agit de penser et de théoriser les changements qui affectent le système touristique. Pour cela nous allons nous appuyer sur la théorie des systèmes sociotechniques proposée par Pierre Dockès (1990). Comme cet auteur précise que les changements affectent le monde, comme certains de ces régions ou pays à partir desquels ils se diffusent, certains secteurs plutôt que d'autres, il est légitime de s'interroger à propos de l'un d'entre eux, en l'occurrence le tourisme, au-delà des spécificités qui le caractérisent (Clergeau et Violier, 2012). Cette analyse énonce que l'alternance entre des phases de forte croissance et des ralentissements dans les cycles économiques longs s'explique par l'émergence assez brutale, ce qui justifie l'usage du mot révolution dans notre titre, puis la stabilité relative qui prévaut ensuite, d'un nouveau paradigme sociotechnique. Si la nouveauté est soulignée par l'affirmation d'innovations techniques, l'auteur insiste bien sur la structuration par le social : « Lors de l'émergence d'un système productif nouveau le changement social est, dès lors, un préalable. » (Dockès, 1990, p. 44). Il nous faudra donc dans notre approche relever les changements techniques et 
économiques qui se font jour, mais également les innovations sociales qui les portent. Le paradigme est donc bien sociotechnique. Il comprend selon Dockès trois dimensions liées : socio-organisationnelle, technique et économique. Nous ajouterons la dimension spatiale qui s'appréhende aux différentes échelles des espaces - dans quelles régions du monde le changement apparait-il et comment se diffuse-t-il ? - et des lieux - quels sont les types de lieux attachés à chaque étape, comme la station et la grande ville, topos du tourisme de masse?

\section{Un nouveau système aujourd'hui ?}

12 Au-delà, il est plus aisé d'appréhender les systèmes du passé que de penser le présent, ce qui est notre ambition. En effet, la théorie n'est ni prédictive ni finaliste, car ce qui advient est le fruit des conflits que l'avènement du nouveau système produit. Il est par conséquent contingent. Cela signifie concrètement que notre analyse d'une révolution en cours, la troisième, ne peut être qu'une proposition partielle puisque rien ne permet d'assurer que la phase de stabilisation ait été atteinte.

Pascal Cuvelier (1998) a déjà tenté d'aborder le tourisme comme un système évolutif et montré les limites d'une approche économique par trop abstraite, qui considère que les formes sont stables dans le temps et dans l'espace et peuvent être traitées avec un nombre limité de concepts clés abstraits. Il a également exposé, en se référant à Robert Salais et Michael Storper (1993) la diversité des modes de production, dans la lignée de Pierre Dockès qui précise qu'un système ne chasse pas l'autre, et que dominant ne signifie en rien exclusif. Le nouveau système induit des recompositions et des mutations qui finalement revivifient en partie les formes anciennes qui ainsi perdurent, comme la révolution industrielle n'a pas entraîné la disparition de l'artisanat mais l'a incorporé.

\section{Lire les transformations du tourisme}

Dès lors notre proposition va consister en une relecture des contributions des historiens qui inclut notamment une hiérarchisation des changements, distinguant parmi les forces en mouvement celles qui relèvent de la sphère sociale et qui seront placées en position motrice par rapport aux changements repérés. Pour ce faire nous construisons un tableau qui distingue les dimensions socio-organisationnelle, technique et économique. À gauche de ce tableau deux colonnes sont consacrées à la périodisation et à la distinction entre période d'émergence et avènement du nouveau système. Une troisième permet d'intégrer le contexte dans lequel se produisent les changements qui affectent le tourisme. D'une part, un système évolue toujours en relation avec son environnement. D'autre part, nous laissons de côté la question de la place du tourisme au sein du système productif capitaliste car c'est un problème difficile à aborder en raison du dualisme qui le caractérise, à la fois moteur et entraîné par l'économie globale, et du discrédit qui pèse sur une pratique sociale jugée futile.

15 L'invention du tourisme, ou la révolution touristique, sur le modèle de la révolution industrielle, doit son émergence, selon Jean Viard (1984), à la dépossession par la bourgeoise industrielle du pouvoir politique qui affecte l'aristocratie au $\mathrm{XVIII}^{\mathrm{e}}$ siècle en Europe occidentale, laquelle se réfugie dans "les vacances", mais nous savons que le terme est davantage utilisé en sociologie que le terme de tourisme qui n'a pas encore 
trouvé ses lettres de noblesse, malgré Saskia Cousin et Bertrand Réau (2009). Mais ce tourisme du petit nombre est caractérisé par des mobilités réservées à une élite qui dispose de beaucoup de temps et qui se déplace essentiellement dans le réseau des résidences de la famille et des connaissances. Les déplacements sont organisés par les voyageurs eux-mêmes en relation avec leur domesticité, et à partir d'informations détenues et livrées par les hôtes ou par le truchement de récits de voyages très volumineux et très peu codifiés (Tissot, 2001).

Les progrès des idées démocratiques sur les terrains sociaux et économiques vont être à l'origine d'un second système fondé sur le grand nombre. L'idée que le progrès doit profiter au plus grand nombre et ne pas être confisqué par une minorité anime des conflits sociaux qui aboutissent, en particulier, à l'effondrement du système du tourisme du petit nombre maîtrisé par les rentiers. La montée des contraintes induite par la complexification de la société industrielle, analysée par Eric Dunning et Norbert Elias (1984) pour comprendre l'invention du sport, peut aussi être mobilisée pour l'avènement de ce nouveau système touristique. L'approfondissement de la révolution industrielle procure également des moyens techniques nouveaux. L'appropriation du chemin de fer par le tourisme - à l'origine l'innovation a été conçue pour transporter des pondéreux des mines vers les usines de transformation -, permet de transférer au secteur touristique la rupture radicale franchie dans les transports. Dès lors puisque plus de monde peut circuler et plus loin, il restait à inventer les lieux pour les accueillir. C'est fait au milieu du siècle. L'invention de l'hôtel qui supplante les infâmes auberges - dont Gustave Flaubert et Maxime Du Camp ne cessent de se plaindre tout au long de leur récit de voyage en Bretagne - et l'invention de la station où l'on passe de quelques unités de villas construites chaque année à des lotissements de plusieurs dizaines (notamment à Arcachon, équipe Mit, 2005), résolvent la question du logement du grand nombre. De manière très exemplaire, c'est le même Émile Pereire qui a créé à Paris le premier véritable hôtel, l'hôtel du Louvre en 1855, et qui a bousculé Arcachon passée sous sa férule entre 1849 et 1852 du stade de petite villégiature bordelaise à celui de station mondaine internationale. Il restera à inventer le camping pour passer de l'émergence du tourisme de masse à son avènement entre les deux guerres mondiales.

Tableau 1 : Lire les transformations du tourisme

\begin{tabular}{|c|c|c|c|c|c|c|}
\hline Système & Phase & Contexte & $\begin{array}{l}\text { Dimension socio- } \\
\text { organisationnelle }\end{array}$ & $\begin{array}{l}\text { Dimension } \\
\text { technique }\end{array}$ & $\begin{array}{l}\text { Dimension } \\
\text { économique }\end{array}$ & $\begin{array}{l}\text { Dimension } \\
\text { spatiale }\end{array}$ \\
\hline \multirow[t]{2}{*}{$\begin{array}{l}\text { 1) invention } \\
\text { du tourisme }\end{array}$} & Émergence & Prétourisme & $\begin{array}{l}\text { Distinction } \\
\text { aristocratique : } \\
\text { voyages et Grand } \\
\text { Tour / loisirs } \\
\text { urbains / } \\
\text { villégiature }\end{array}$ & $\begin{array}{l}\text { Hébergement dans } \\
\text { les auberges ou } \\
\text { châteaux de la } \\
\text { famille et des amis }\end{array}$ & & $\begin{array}{l}\text { Pas d'empreinte } \\
\text { spatiale } \\
\text { marquée; } \\
\text { itinéraires peu } \\
\text { nombreux et } \\
\text { intra- } \\
\text { européens }\end{array}$ \\
\hline & Avènement & $\begin{array}{l}\text { Révolution } \\
\text { industrielle }\end{array}$ & $\begin{array}{l}\text { Autonomie des } \\
\text { touristes mais } \\
\text { petite frange de la } \\
\text { société }\end{array}$ & $\begin{array}{l}\text { Villégiature; } \\
\text { touristification des } \\
\text { villes thermales }\end{array}$ & $\begin{array}{l}\text { Pas de logique } \\
\text { économique ; } \\
\text { autoproduction }\end{array}$ & $\begin{array}{l}\text { Quelques villas } \\
\text { peu } \\
\text { nombreuses }\end{array}$ \\
\hline
\end{tabular}




\begin{tabular}{|c|c|c|c|c|c|c|}
\hline \multirow[b]{2}{*}{$\begin{array}{l}\text { 2) tourisme } \\
\text { de masse }\end{array}$} & $\begin{array}{l}\text { Émergence: } \\
\text { le grand } \\
\text { nombre } r \text { à } \\
\text { partir de la } \\
\text { fin du } \text { XIX }^{\mathrm{e}} \\
\text { siècle }\end{array}$ & $\begin{array}{l}\text { Effets plus } \\
\text { positifs de la } \\
\text { révolution } \\
\text { industrielle; } \\
\text { avènement du } \\
\text { grand nombre; } \\
\text { colonisation du } \\
\text { monde par les } \\
\text { Européens }\end{array}$ & $\mid \begin{array}{ll}\text { Élargissement } & \\
\text { social } & \text { et } \\
\text { apparition de } & \text { la } \\
\text { coproduction } & \end{array}$ & $\begin{array}{l}\text { Guide touristique; } \\
\text { hôtellerie; } \\
\text { apparition du tour- } \\
\text { operating } \\
\text { (assemblage); } \\
\text { catalogue; } \\
\text { chemin de fer } \\
\text { détourné }\end{array}$ & $\begin{array}{lr}\text { Affirmation d'une } \\
\text { logique } \\
\text { économique; } \\
\text { organisation des } \\
\text { acteurs } \\
\text { tourisme }\end{array}$ & $\begin{array}{l}\text { Invention de la } \\
\text { station ou resort } \\
\text { comptoir, } \\
\text { club; sortie de } \\
\text { l'Europe } \\
\text { (Égypte, tours } \\
\text { du monde); } \\
\text { diffusion dans } \\
\text { les colonies de } \\
\text { peuplement ( } \\
\text { hill stations) }\end{array}$ \\
\hline & $\begin{array}{l}\text { Avènement: } \\
\text { États-Unis c. } \\
1920 \text {; Europe } \\
\text { c. } 1950\end{array}$ & $\begin{array}{l}\text { Consommation } \\
\text { de masse }\end{array}$ & $\begin{array}{ll}\text { Société } & \text { de } \\
\text { consommation de } & \\
\text { masse } ; & \\
\text { généralisation des } \\
\text { congés payés }\end{array}$ & $\begin{array}{l}\text { Automobile grand } \\
\text { public, avion, } \\
\text { caravane; } \\
\text { camping; } \\
\text { structuration de } \\
\text { l'offre; } \\
\text { industrialisation de } \\
\text { l'offre }\end{array}$ & $\begin{array}{l}\text { Dualisme } \\
\text { MégaTO/ TPE } \\
\text { hyperspécialisées; } \\
\text { logique } \\
\text { d'économie } \\
\text { d'échelle sur la } \\
\text { production des } \\
\text { services dans la } \\
\text { distribution }\end{array}$ & $\begin{array}{l}\text { Métropoles } \\
\text { touristiques et } \\
\text { mégastations; } 1 \\
\text { ère } \\
\text { mondialisation } \\
\text { du tourisme; } \\
\text { diffusion } \\
\text { spatiale dans le } \\
\text { monde mais à } \\
\text { partir des } \\
\text { foyers et } \\
\text { européens et } \\
\text { des pays neufs }\end{array}$ \\
\hline \multirow[t]{2}{*}{$\begin{array}{l}\text { 3) } 3^{\mathrm{e}} \\
\text { révolution } \\
\text { industrielle }\end{array}$} & $\begin{array}{l}\text { Émergence : } \\
\text { fin du } \mathrm{xx}^{\mathrm{e}} \\
\text { siècle }\end{array}$ & Individualisation & $\begin{array}{lr}\text { Remise } & \text { en } \\
\text { question } & \text { du } \\
\text { tourismer } & \text { de } \\
\text { masse : tourisme } & \\
\text { durable, } & \\
\text { écotourisme...; } \\
\text { réalisation } \\
\text { personnelle; } \\
\text { coproduction/ } \\
\text { cocréation }\end{array}$ & 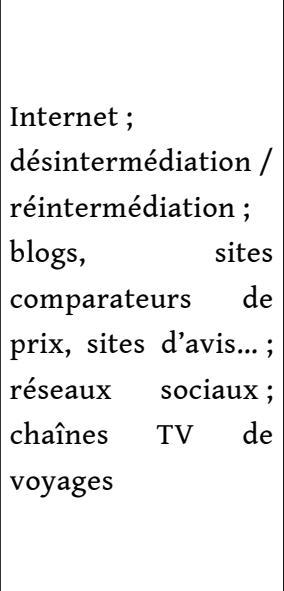 & $\begin{array}{l}\text { Fin du mégaTO?; } \\
\text { passage } \\
\text { d'économies } \\
\text { d'échelles sur la } \\
\text { conception à des } \\
\text { économies } \\
\text { d'échelles sur la } \\
\text { distribution?; } \\
\text { modèle low-cost }\end{array}$ & $\begin{array}{l}\text { Adaptation des } \\
\text { lieux et mise en } \\
\text { tourisme des } \\
\text { marges; } 2^{\mathrm{e}} \\
\text { mondialisation : } \\
\text { accès au } \\
\text { tourisme des } \\
\text { sociétés } \\
\text { émergentes; de } \\
\text { nouveaux } \\
\text { foyers } \\
\text { touristiques } \\
\text { apparaissent }\end{array}$ \\
\hline & Avènement & & & & & \\
\hline
\end{tabular}

\section{La troisième révolution touristique}

Dès lors le système touristique actuel change, c'est presque une évidence. Mais pourquoi et en quoi est-il radicalement modifié, de manière à justifier l'appellation de troisième révolution touristique ${ }^{2}$ ? 


\section{Un tourisme devenu vraiment mondial}

18 Les premiers signes viennent des mutations qui affectent le domaine socioorganisationnel. Il nous semble que deux traits majeurs apparaissent. D'une part, le monde actuel est marqué par un double mouvement de mise en tourisme total. Premièrement, le monde entier est devenu touristique. En effet, les touristes s'insinuent partout. L'œcoumène touristique est devenu plus vaste que le mode sédentaire. Des formes qui se revendiquent d'un tourisme différent, durable, écologique ou solidaire, ne contribuent finalement qu'à étendre son emprise. Deuxièmement, dans les régions les plus touristiques, des processus d'intensification sont à l'œuvre qui transforment en objets touristiques des réalités autres, comme les mines, les usines, les studios de cinéma... Enfin, la diffusion sociale du tourisme se poursuit par le truchement de dynamiques sociales, économiques et techniques. Les générations nées ou actives pendant l'avènement du tourisme de masse arrivent aujourd'hui aux âges avancés de la retraite. Elles entendent continuer à être touristes, si bien que l'ancienne dichotomie qui se manifestait par un faible taux de départ des personnes âgées a aujourd'hui quasiment disparu, et la fin du tourisme, pour chacun, est reportée aux très grands âges. Plusieurs techniques sont mobilisées par les acteurs pour maîtriser les coûts et poursuivre les gains par les économies d'échelle qui affectent aujourd'hui la distribution (Clergeau et alii, 2014). De même, la dynamique low-cost affecte le transport aérien. La rationalisation de l'organisation et le renoncement à un esprit élitiste se conjuguent pour abaisser les tarifs pour les moyennes distances, celles qui justement concernent la majeure partie des touristes transportés par ce mode.

Ensuite, l'accès au tourisme des sociétés émergentes manifeste la poursuite de la diffusion sociétale du tourisme. Mais alors que cette tension s'est effectuée jusqu'à présent au sein des sociétés occidentales, elle les dépasse largement aujourd'hui. Le développement économique qui affecte les sociétés non occidentales ${ }^{3}$, qui s'apparente à un rattrapage, est le produit d'un rejet de la domination du monde par les métropoles coloniales et l'affirmation de la volonté des peuples de vivre mieux. Il s'agit d'un processus inégalement engagé dans le monde. Il a débuté au Japon dans les années 1960, s'est diffusé dans l'orbite économique de ce pays dans les années 1980 avant d'affecter aujourd'hui les pays très peuplés comme la Chine, l'Inde, l'Indonésie, le Brésil... Il est suffisamment approfondi pour contribuer à la dynamique du secteur touristique. Par ailleurs, comme la rugosité de la distance continue à participer à l'organisation des flux, puisque $85 \%$ des individus inscrivent leurs mobilités touristiques dans leur continent, ce processus de diffusion sociétale du tourisme participe à la finitude touristique du monde. Les régions proches des nouveaux foyers émetteurs sont principalement concernées par la mise en œuvre de pratiques pour une part nouvelles et que pour une autre les nouvelles sociétés touristiques s'approprient (Sacareau et alii, 2015 ; Violier et Giffon, 2015).

20 Par ailleurs, dans les pays anciennement touristiques, la remise en question du système précédent aboutit à son démantèlement progressif. Le succès du tourisme de masse est tel que dans les pays les plus riches, des taux de $70 \%$ de la population qui y accède dans une année sont atteints, alors que $15 \%$ y renoncent par choix, ne laissant finalement que $15 \%$ de laissés pour compte, même si cela demeure insupportable pour les exclus. Mais ces résultats sont à peine atteints que déjà les critiques fusent. Accusé de tous les maux, d'être standardisé - alors que son succès repose en partie sur cette technique -, d'être 
destructeur pour l'environnement - alors qu'il n'est pas démontré que la concentration est moins efficace que la dispersion dans la gestion de l'environnement, comme si par exemple l'étalement urbain était plus pertinent du point de vue de la gestion des transports -, de profiter aux acteurs professionnels plus qu'aux habitants alors que la liste des pays qui ont assis leur décollage économique sur le tourisme est longue... bref, remis en question de toute part, le tourisme évolue. En fait, travaillé par le courant structurant de l'affirmation de l'individu, le tourisme passe du tourisme de masse au tourisme de masse personnalisé. Dès lors se diffusent des pratiques nouvelles ou renouvelées à tel point que la diversification des modes d'être touriste interpelle et justifie en retour un questionnement sur le sens même du tourisme. Au-delà du constat que cette difficulté à cerner l'objet n'est pas nouvelle (Michel, 1997), notre société demeure structurée par le dualisme entre temps de travail et loisir, et le tourisme demeure une mobilité inscrite dans cette division.

\section{De nouvelles relations entre les acteurs}

21 Or cette évolution sociale, la personnalisation des pratiques, a lieu parce qu'Internet permet aux acteurs économiques de gérer à la fois les grands nombres et la diversité des demandes comme l'affirmation de l'autonomie, qui se traduit dans le tourisme par l'approfondissement de la participation des individus à la production. En particulier, l'assemblage est de moins en moins le fait des seuls tour-opérateurs et de plus en plus l'œuvre des touristes eux-mêmes. Dès lors les acteurs économiques sont amenés à reconsidérer leurs fonctions et leurs modèles économiques. Comme avec le chemin de fer au milieu du XIX ${ }^{e}$ siècle, un saut technologique majeur né en dehors est incorporé dans le système touristique et le transforme. À nouveau, les innovations sont portées par des créateurs d'entreprises et des acteurs issus d'autres univers économiques qui bousculent les rôles établis.

Cependant, dans les pays anciennement touristiques, le système induit la recomposition des acteurs. Le circuit ou le séjour standardisés se spécialisent sur les segments les moins transformés par la montée de l'individu, à savoir les touristes les moins enclins à pratiquer la personnalisation en raison de leur faible capital sous toutes ses formes (touristique, mobilitaire, économique, social, culturel), qu'ils résident dans les pays avancés ou émergents. Mais ces formes sont aussi traversées par des innovations sous la pression des individus âgés qui, voulant continuer à être touristes, exercent des pressions sur les acteurs professionnels qui cherchent à innover, conscients qu'ils sont du poids économique croissant des retraités. Ainsi l'autocar est-il revisité comme mode de transport et propose des conditions de confort meilleures et des services nouveaux, comme le pré-acheminement qui épargne aux personnes âgées les affres des déplacements préliminaires vers les hubs. Au-delà, recourir aux services d'un touropérateur est un choix doté d'une certaine rationalité, soit lorsque les individus n'ont pas le temps ou l'énergie à consacrer à organiser leur voyage, soit lorsque le degré d'altérité risque de soumettre les corps et les esprits à des conditions difficiles qui ne seront pas sans effets. Au lieu de lire dans les voyages organisés une démission du touriste et une soumission à une organisation tentaculaire, il nous semble plus judicieux d'interpréter un choix au sein d'une marge de manœuvre et de tenter d'en comprendre les ressorts. Si Pascal Cuvelier (1998) met en avant la simplicité de la proposition et le désir d'autonomie plus ou moins grand porté par les individus, selon la grille de lecture des économistes 
(offre et demande) nous avons déjà développé le fait que la diversité du monde devait également être mobilisée (équipe Mit, 2002; Mondou et Violier, 2009) comme les compétences des touristes (Ceriani et alii, 2008 ; Brougère, 2012, 2014).

\section{Hybridation des pratiques}

23 Ces évolutions font que, dans les hauts lieux où sont coprésents des touristes issus des différentes sociétés du monde, des manières d'habiter le monde très contrastées sont rendues visibles. Les individus fortement dotés en capital et en compétences, appartenant à des sociétés anciennement touristiques, qui circulent selon des pratiques relativement auto-organisées, se donnent à voir aux individus des sociétés émergentes. La confrontation entre les différents modes informent sur les possibles et questionnent le voyage en groupe, technologie par laquelle le tourisme est découvert et appris. La proposition de Gilles Brougère (2012) selon laquelle l'apprentissage du tourisme passe par la coprésence dans des équipements touristiques, comme les villages de vacances au sein desquels se développe un apprentissage informel, peut être étendue aux hauts lieux touristiques.

Le système touristique évolue aussi en interrelation avec des valeurs qui émergent dans son environnement et qui le questionnent. Il en est ainsi du développement durable apparu au début des années 1970, qui inspire un renouvellement des pratiques et des stratégies (Knafou, 2003; Knafou et Pickel Chevalier, 2011; Pickel Chevalier, 2014). Également, les manières d'être touriste inventées dans les autres mondes vont se diffuser dans les sociétés anciennement touristiques. Cette proposition mérite sans doute d'être travaillée mais des indices permettent de l'énoncer. Nous retiendrons deux exemples. Le parc naturel inventé en Amérique du Nord dans la seconde moitié du XIXe siècle s'est ensuite constitué en modèle et s'est diffusé à travers le monde. Le ski nocturne apparaît comme une contribution de la société japonaise au tourisme.

\section{Conclusion}

Loin d'être moribond, comme l'affirment les tenants de la « fin du tourisme », ou dépassé, comme le laisse penser l'expression " post-tourisme ", le tourisme manifeste au contraire une dynamique de renouvellement dans un contexte qui demeure marqué par la division des temps issu de la révolution industrielle. Cette évolution est plurielle. Tout d'abord, l'inépuisable imagination des humains invente et réinvente des manières d'être touriste. Ensuite, l'ensemble du monde est devenu mobile (Stock, 2008). Et cet avènement d'un tourisme de masse à l'échelle planétaire induit un autre renouvellement qui agite les pratiques touristiques à travers les processus d'invention, d'imitation, d'hybridation, entre les sociétés des différents mondes.

L'approche systémique traduit d'une part l'idée que la révolution touristique est une mutation qui affecte différents éléments en interrelation et prend donc la forme de processus qui se produisent dans un temps moyen et non d'un seul fait pris isolément et précisément daté. D'autre part se produit une recomposition de l'existant qui ne disparait pas. Ainsi le voyage organisé standardisé tend à être relativisé dans les métropoles, tandis qu'il constitue le mode conquérant des sociétés émergentes. 
extes présentés dans ce numéro hors-série de la revue Mondes du tourisme explorent cette interrogation sur la dynamique du système touristique. Ils sont présentés en deux parties, l'une est organisée autour de l'entrée par la diversification des pratiques des touristes et par les stratégies déployées par les acteurs professionnels ; l'autre est abordée par les lieux et les territoires.

\section{BIBLIOGRAPHIE}

Isabelle Babou et Philippe Callot, Les Dilemmes du tourisme, « Gestion internationale », Vuibert, 2007.

Isabelle Babou et Philippe Callot, Que serait le tourisme sans pétrole?, « Tourismes et sociétés », L'Harmattan, 2012.

Philippe Bourdeau, «Interroger les mutations et recompositions en cours », dans Hugues François, Alain Bourdeau et Liliane Perrin-Bensahel (dir.), Fin (?) et confins du tourisme. Interroger le statut et les pratiques de la récréation contemporaine, "Les idées et les théories à l'épreuve des faits ", L'Harmattan, 2013, p. 17-39.

Marc Boyer, L'Invention du tourisme, « Découvertes », Gallimard, 1996.

Gilles Brougère, «Pratiques touristiques et apprentissages ", Mondes du Tourisme, $\mathrm{n}^{\circ}$ 5, 2012, p. 62-75 [http://tourisme.revues.org/415].

Gilles Brougère et Giulia Fabiano (dir.), Apprentissages en situation touristique, « Éducation et didactique », Presses universitaires du Septentrion, 2014.

Christine Cabasset-Semedo, Emmanuelle Peyvel, Isabelle Sacareau et Benjamin Taunay, « De la visibilité à la lisibilité : le tourisme domestique en Asie ", Espace populations sociétés n ${ }^{\circ} 2-3,2010$, p. 231-235 [http://eps.revues.org.buadistant.univ-angers.fr/4118].

Giorgia Ceriani, Vincent Coëffé, Jean-Christophe Gay, Rémy Knafou, Mathis Stock, Philippe Violier, « Conditions géographiques de l'individu contemporain », Espaces Temps, 2008 [http:// www.espacestemps.net/articles/conditions-geographiques-individu-contemporain/].

Cécile Clergeau et Philippe Violier, « Le concept de cluster est-il soluble dans le tourisme? ", Téoros, vol. 31, $\mathrm{n}^{\circ} 2,2012$, p. 60-71.

Cécile Clergeau (dir.), Olivier Galsberg, Philippe Violier (coll.), Management des entreprises du tourisme. Stratégie et organisation, Dunod, 2014.

Saskia Cousin et Bertrand Réau, Sociologie du tourisme, « Repères », La Découverte, 2009.

Pascal Cuvelier, Anciennes et nouvelles formes de tourisme: une approche socio-économique, « Tourismes et sociétés », L'Harmattan, 1998.

Jean-Michel Dewailly, « L'espace récréatif : du réel au virtuel ? », Espace géographique, vol. 26, n 26-3, 1997, p. 205-213.

Pierre Dockès, « Formation et transferts des paradigmes socio-techniques ", Revue française d'économie, vol. 5, n 4, 1990, p. 29-82. 
Eric Dunning et Norbert Elias, Sport et civilisation : la violence maîtrisée, Fayard, 1994.

Équipe Mit, Tourismes. 1, Lieux communs, « Mappemonde », Belin, 2002.

Équipe Mit, Tourismes. 2, Moments de lieu, « Mappemonde », Belin, 2005.

Équipe Mit, Tourismes. 3, La révolution durable, « Mappemonde », Belin, 2011.

Hans Magnus Enzensberger, « Eine Theorie des Tourismus », Merkur, nº 12, 1958.

Julien Gardaix, « Tourisme et mondialisation : vers de nouvelles pratiques ? ", dans Hugues François, Alain Bourdeau et Liliane Perrin-Bensahel (dir.), Fin (?) et confins du tourisme. Interroger le statut et les pratiques de la récréation contemporaine, « Les idées et les théories à l'épreuve des faits », L'Harmattan, 2013, p. 163-175.

Alain Girard, «Faut-il raccorder une théorie générale de la post-modernité à une théorie de moyenne portée du post-tourisme ? », dans Hugues François, Alain Bourdeau et Liliane PerrinBensahel (dir.), Fin (?) et confins du tourisme. Interroger le statut et les pratiques de la récréation contemporaine, «Les idées et les théories à l'épreuve des faits ", L'Harmattan, 2013, p. 43-52.

Erdman Gormsen, «The spatio-temporal development of international tourism. Attempt at the centre periphery model ", dans La Consommation d'espace par le tourisme et sa préservation, Centre des hautes études touristiques, 1981, p. 150-170.

Jafar Jafari, « Le système du touriste : modèles socio-culturels en vue d'applications théoriques et pratiques », Society and Leisure, vol. 11, n 1, 1988.

Rémy Knafou, « Vers un tourisme responsabilisé », dans Mathis Stock (dir.), Le Tourisme : acteurs, lieux, enjeux, «Belin Sup. Géographie », Belin, 2003, p. 213-258.

Rémy Knafou et Sylvine Pickel Chevalier, « Tourisme et "développement durable" : de la lente émergence à une mise en œuvre problématique », Géoconfluences, 2011 [http:// geoconfluences.ens-lyon.fr/doc/typespace/tourisme/TourScient4.htm].

Rémy Knafou et Mathis Stock, 2003, « Tourisme », dans Jacques Lévy et Michel Lussault (dir.), Dictionnaire de la géographie, Belin, 2003, p. 931-934.

Franck Michel, Tourisme, culture et modernité en pays Toraja : Sulawesi-Sud, Indonésie, « Tourismes et sociétés », L’Harmattan, 1997.

Équipe Mit, Tourismes. 1, Lieux communs, « Mappemonde », Belin, 2002.

Équipe Mit, Tourismes. 2, Moments de lieu, « Mappemonde », Belin, 2005.

Équipe Mit, Tourismes. 3, La révolution durable, « Mappemonde », Belin, 2011.

Véronique Mondou et Philippe Violier, « Projets, pratiques et lieux touristiques, quelles relations ?», Mappemonde, nº 94, 2009 [http://mappemonde.mgm.fr/num22/articles/art09201/ html].

Asep Parantika, Le Tourisme domestique indonésien. Entre transfert et innovation, vers un nouveau modèle?, thèse de l'université d'Angers, 2015.

Asep Parantika et Sylvine Pickel Chevalier, « Expériences touristiques et rapport au corps sur les littoraux en Indonésie ", dans Jean-Michel Decroly (dir.), Le Tourisme comme expérience. Regards interdisciplinaires sur le vécu touristique, Presses de l'université du Québec, 2015, p. 197-202.

Sylvine Pickel Chevalier, L'Occident face à la nature, de l'Antiquité à nos jours : à la confluence des sciences, de la philosophie et des arts, « Idées reçues », Le cavalier bleu, 2014.

Bertrand Réau, Les Français et les Vacances, CNRS, 2011. 
Isabelle Sacareau, «La diffusion du tourisme dans les sociétés non occidentales : entre imitation et hybridation, l'exemple de la fréquentation de la montagne himalayenne par les touristes indiens ", Mondes du tourisme, hors-série « Tourisme et mondialisation », 2011, p. 310-317.

Isabelle Sacareau, Benjamin Taunay et Emmanuelle Peyvel (dir.), La Mondialisation du tourisme. Les nouvelles frontières d'une pratique, « Espace et Territoires », Presses universitaires de Rennes, 2015.

Robert Salais et Michael Storper, Les Mondes de production. Recherche sur l'identité économique de la France, Éditions de l'EHESS, 1993.

Miguel Ségui Llinas, Les Nouvelles Baléares. La rénovation d'un espace touristique mythique, « Tourismes et sociétés », L’Harmattan, 1995.

Mathis Stock (dir.), Le Tourisme : acteurs, lieux, enjeux, « Belin Sup. Géographie », Belin, 2003.

Mathis Stock, «L'hypothèse de l'habiter poly-topique : pratiquer les lieux géographiques dans les sociétés à individus mobiles », Espaces Temps, 2006, [http://www.espacestemps.net/articles/ hypothese-habiter-polytopique/]

Mathis Stock, «Il mondo é mobile », dans Jacques Lévy (dir.), L'Invention du monde : une géographie de la mondialisation, Presses de la Fondation des sciences politiques, 2008.

Benjamin Taunay, Le Tourisme intérieur chinois, « Espace et territoires », Presses universitaires de Rennes, 2011.

Laurent Tissot, Naissance d'une industrie touristique. Les Anglais et la Suisse au XIX ${ }^{e}$ siècle, « Histoire ", Payot, 2001.

John Urry, Consuming Places, Routledge, 1995.

Jean Viard, Penser les vacances, Actes Sud, 1984.

Philippe Violier (dir.), Le Tourisme, un phénomène économique, « Études », La Documentation française, 2013.

Philippe Violier, «Thierry Paquot, Le Voyage contre le tourisme », Mondes du tourisme, $\mathrm{n}^{\circ} 10$, 2014, p. 103-104 [http://tourisme.revues.org/391].

Philippe Violier et Sigrid Giffon, « Voyages dans le monde : une figure de l'habiter mobile », L'Information géographique, $\mathrm{n}^{\circ}$ 3, 2015, p. 17-33.

\section{NOTES}

1. Le voyage est encore mobilisé soit par des intellectuels élitistes qui peinent à penser le tourisme et se réfugient dans le mépris, bien qu'ils s'en défendent, à titre d'exemple Thierry Paquot dans Le Voyage contre le tourisme, 2014, voir notre recension (Violier, 2014); soit par une partie de la sphère des acteurs du tourisme, touristes ou entrepreneurs, qui naviguent entre distinction et actions de marketing.

2. Le choix du mot "révolution» repose sur le constat qu'à un moment donné plusieurs changements de grande ampleur se produisent dans un temps relativement rapproché, même si des éléments du système précédent persistent sans modifications majeures et sont incorporés dans le nouveau système.

3. Le débat suscité par l'expression «monde occidental » nous semble justifier l'usage de la périphrase "les sociétés occidentales", et a fortiori celle des "sociétés non occidentales », qui présentent l'avantage de souligner de manière dialogique l'unité et la diversité de ces ensembles. 


\section{RÉSUMÉS}

Cette contribution propose un cadre conceptuel pour penser l'évolution du tourisme. En mobilisant les travaux des historiens, elle réaffirme l'historicité du tourisme niée par certains auteurs, à savoir que le tourisme n'a pas toujours existé et qu'il est apparu à un moment de la civilisation occidentale, comme un effet et un besoin de la civilisation industrielle. C'est la première révolution qui accouche du tourisme. Le tourisme a ensuite évolué, passant par plusieurs états systémiques avant de passer au tourisme de masse, signe d'une nouvelle révolution. Enfin, une troisième révolution touristique peut être observée à travers un certain nombre de ruptures d'aujourd'hui : passage d'un tourisme occidental à un stade réellement mondial, développement de pratiques plus personnalisées en rupture avec les formes standardisées, émergence de nouveaux acteurs... Dans cette évolution nous identifions les forces sociales comme le moteur du changement, plutôt que les techniques qui sont des moyens mobilisés par les individus et les sociétés pour renouveler les formes du tourisme.

This paper proposes a conceptual framework to understand how tourism evolves. By using the work of historians it reaffirms the historicity of tourism rejected by some authors, in the sense that tourism has not always existed and that it appeared in parallel with western civilization as an effect and a need of industrial society. This was the first revolution that led to tourism and has subsequently evolved, transiting several systemic states before the move to mass tourism as a new revolution. Finally, a third tourist revolution can be identified by the changes we are witnessing today: the switch from Western tourism to a truly global one, the development of more personalized activities, a break from standardized formulas and the emergence of newcomers to the industry, etc. In this evolution we will reveal that social forces are powering this change, rather than techniques which are used by individuals and societies to regenerate different types of tourism.

\section{INDEX}

Mots-clés : dynamique du tourisme, révolution touristique, système touristique, tourisme de masse

Keywords : tourism dynamic, tourism revolution, tourism system, mass tourism

\section{AUTEUR}

\section{PHILIPPE VIOLIER}

Professeur de géographie à l'université d'Angers, Esthua. 